\title{
A look at the post-harvest management of fruits
}

Fifty years ago, issues 7 and 8 of volume 15 of the Fruits journal placed an emphasis on post-harvest management of fruits.

The first article by C. Leblond was on Preserving fruit in controlled atmosphere enclosures bounded by plastic films and already showed the many benefits offered by this promising technique and the interest in "the use of boxes with walls selectively permeable to gas".

A second article by P. Dupaigne dealt with the Determination of the percentage of pulp in fruit juices and vegetables; it was intended to pave the way for a better standardization of products for industrial use.

A third article by J.J. Doesburg and L. De Vos was interested in new technological processes based on Mixed pasteurized fruit juice and milk for a prolonged storage.

Lastly, leaving aside the technical aspects, the fourth article highlighted the benefit of addressing the Future opportunities for the orange trade in Europe. The author, M.R. Cadillat, was referring to a century-old but still relevant citation from the English philosopher Thomas Carlyle: "Since a while bumanity has reached a stage whereby the production is nothing. What is key is to ensure a rational distribution"; he ended on a philosophical note, writing "Of course we should not delude ourselves. If reality is anything but the bope of men, future is for the optimists as optimism is synonymous with vitality and good will".

\section{Lu dans Fruits, il y a 50 ans...}

\section{Regards sur l'après-récolte des fruits}

Il y a 50 ans, les numéros 7 et 8 du volume 15 de la revue Fruits donnaient une place importante au devenir des fruits après la récolte.

Un premier article de C. Leblond portait sur la Conservation des fruits en atmosphère contrôlée dans des enceintes limitées par des films de matière plastique et montrait déjà les nombreux avantages offerts par cette technique prometteuse et l'intérêt de "l'emploi de caissons à parois sélectivement perméables aux gaz ".

Un deuxième article de $\mathrm{P}$. Dupaigne traitait de la Détermination du pourcentage de pulpe dans les jus de fruits et de légumes dont l'objectif était d'ouvrir la voie à une meilleure normalisation des produits pour leur usage industriel.

Un troisième article de J.J. Doesburg et L. De Vos s'intéressait à de nouveaux procédés technologiques basés sur des Mélanges pasteurisés de jus de fruits et de lait, destinés a un stockage prolongé.

Enfin, sortant des aspects techniques, un quatrième article montrait tout l'intérêt de se pencher sur les Possibilités d'avenir du commerce des oranges en Europe. L'auteur, R.M. Cadillat, se référait à une citation du philosophe anglais Thomas Carlyle, vieille d'un siècle mais toujours d'actualité, selon laquelle "Depuis longtemps l'bumanité est arrivée au stade grâce auquel la production n'est plus rien. Ce qui est tout, c'est d'assurer une distribution rationnelle"; il terminait sur une note philosophique en écrivant "Certes il ne faut pas se leurrer. Si la réalité est tout autre que l'espoir des hommes, l'avenir est aux optimistes autant qu'optimisme est synonyme de dynamisme et de bon vouloir". 